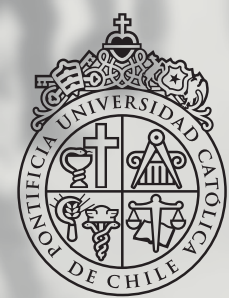

PONTIFICIA

UNIVERSIDAD

CATÓLICA

DE CHILE
Revista

Trabajo
Análisis crítico

de la teoría de cambio en intervenciones de visitas domiciliarias

en el programa

Chile Crece Contigo

Kenichi Haramoto

Paula Verdugo

Visita domiciliaria en Trabajo Social:

¿cómo la valoran y utilizan un grupo de estudiantes de la profesión?

Paula Chamorro

Alicia Razeto
Propuesta de un modelo de supervisión reflexiva de visitas domiciliarias realizadas en un programa dirigido a familias de recién nacidos

María Carolina Velasco Hodgson

Daniela Tuma IBIETA

Claudia Aldana Pereira

\section{Formación continua en} visita domiciliaria.

Análisis cualitativo de una metasupervisión reflexiva

Claudia Cerfogli

Paulina Contreras

Experiencias sobre el uso de eHealth en educación para la salud, gestación y primera infancia:

una revisión bibliográfica

Benjamin Charles Nielsen Guzmán 



\title{
Análisis crítico de la teoría de cambio en intervenciones de visitas domiciliarias en el programa Chile Crece Contigo
}

\author{
Critical analysis of the theory of change used in Home \\ Visiting interventions in the Chile Crece Contigo Program
}

\author{
Kenichi Haramoto \\ Psicólogo Pontificia Universidad Católica de Chile. Escuela de Psicología. kharamot@uc.cl \\ Paula Verdugo \\ Psicóloga Pontificia Universidad Católica de Chile. Escuela de Psicología.pmverdug@uc.cl
}

\begin{abstract}
Resumen
Las experiencias nacionales de visitas domiciliarias, como estrategia de intervención en programas de salud, han tenido impactos limitados. No existe un consenso sobre las causas del bajo impacto que han tenido este tipo de intervenciones, a pesar que el potencial de las visitas domiciliarias ha sido confirmado en estudios internacionales. En Chile faltan estudios que permitan analizar qué sucede, concretamente, durante las visitas. Estos estudios descriptivos, llamados de "caja negra", permiten entender lo que sucede durante una intervención de visitas domiciliarias. En el contexto del programa Chile Crece Contigo, se ha realizado un primer estudio de "caja negra" sobre las intervenciones de visitas domiciliarias. Este artículo busca resaltar la importancia que han destacado otros estudios internacionales sobre la teoría de cambio o modelo explicativo en el éxito o impacto de la intervención, basándose en análisis de casos y extractos de las visitas. El programa Chile Crece Contigo busca generar cambios en el proceso de gestación, salud y educación en la infancia temprana. Hasta el momento, la teoría de cambio ha estado basada en un modelo pedagógico. Secundariamente, este artículo presenta como alternativa un modelo de intervenciones basados en la Entrevista Motivacional desarrollada por Miller y Rollnick (1999).
\end{abstract}

Palabras clave: Visita domiciliaria, entrevista motivacional, cambio, salud pública, formación.

\begin{abstract}
The use of home visiting as an intervention in national programs has shown very limited impact. There is no agreement on why these interventions have been unsuccessful given that they have shown great potential at a global level through international studies. Chile is in need of studies that show what happens inside a home visit and how it really develops. These descriptive-type studies have been named "black-box" studies, and they allow access to the unknown world of the visit through recordings and transcripts. Chile Crece Contigo program has recently developed the first "black-box" study of Home Visiting interventions. The goal of this article is to highlight the role of the theory of change or theoretical framework on which these interventions are designed, and how some frameworks provide better results for the improvement of health and education in early childhood. One of these frameworks is the method called Motivational Interviewing developed by Miller y Rollnick (1999).
\end{abstract}

Keywords: Home visiting, motivational interviewing, change, healthcare, training.

\section{Introducción}

Los programas de visitas domiciliarias en edad temprana se ofrecen con el propósito de crear un ambiente seguro y propicio para el desarrollo y la salud de recién nacidos e infantes, así como también en la prevención del abuso y la negligencia infantil (Segal, Sara Opie, \& Dalziel, 2012; Sweet $\&$ Appelbaum, 2004). En la actualidad, las visitas domiciliaras constituyen una de las estrategias más prometedoras en la intervención temprana con mujeres embarazadas, infantes y sus familias (Kotliarenco, Gómez, Muñoz, \& Aracena, 2010). 
Sin embargo, este formato de intervención ha sido foco de controversias porque ha tenido resultados heterogéneos, en ocasiones, no alcanzando los resultados esperados (Gomby, 2007; Hebbeler \& Gerlach-Downie, 2002; Korfmacher et al., 2008; Sweet \& Appelbaum, 2004).

Frente a la disyuntiva entre la potencialidad de las visitas domiciliarias y su bajo impacto, Kathleen Hebbeler y Suzanne Gerlach-Downie (2002) realizan el primer estudio llamado "de caja negra". En éste, investigan con cámaras de video y grabaciones el desempeño de los monitores que realizan las visitas domiciliarias para confirmar la implementación del programa en todos sus niveles. La conclusión de este estudio confirma la potencialidad de las visitas domiciliarias, mostrando que la falla se encuentra en la forma de conceptualizar los programas y las teorías de cambio que los sustentan. Korfmacher et al. (2008) construyen sobre esta tesis y plantean la importancia de articular la intervención con cambios reales en la relación entre los infantes y los padres. A nivel global, los programas de visitas domiciliarias han reportado resultados de carácter mixto, siendo algunos positivos, otros neutrales y algunos negativos dependiendo de los objetivos que pretenden alcanzar (LeCroy \& Whitaker, 2005). Se muestra, también, que hay una baja efectividad en los programas de mediano y largo plazo que no tienen una teoría de cambio definida (Aracena et al., 2013; Kendrick, 2000).

La teoría del cambio ha sido definida como un conjunto de declaraciones sobre el cómo y el por qué se espera que una iniciativa funcione y lo que se necesita para alcanzar los resultados deseados (Weiss, 1995). Hebbeler y Gerlach-Downie (2002) describen que la teoría de cambio ha sido utilizada para describir las metas, métodos y los supuestos en los que se basa el programa social. En otras palabras, no sólo se refiere a un modelo teórico, sino que también al método práctico para alcanzar las metas. Estos autores destacan la importancia de que tanto el diseño como los monitores que implementan el programa tengan una visión consistente respecto a cómo se impacta la salud y el bienestar de los infantes. En resumen, la pregunta sobre la teoría de cambio se relaciona directamente con el método de la intervención, lo que se retomará más adelante en el artículo mediante la metodología de entrevistas motivacionales.

Sobre el método pedagógico, la crítica principal sobre las visitas domiciliaras dirigidas al mejoramiento de la salud y desarrollo infantil ha sido que éste consiste en el aprendizaje de un contenido re- lacionado con la crianza y la maternidad (Hebbeler $\&$ Gerlach-Downie, 2002). Las autoras critican que, detrás de este método, hay una teoría de cambio que asume una relación proporcional entre el mayor conocimiento sobre desarrollo y crianza, con cambios efectivos en el bienestar de los infantes. Ésta sería la causa de la baja efectividad de los programas, porque aprender este contenido no genera cambios en el estado de los niños, necesariamente. En respuesta a la baja efectividad de una metodología exclusivamente pedagógica se ha propuesto complementar el contenido educativo con el desarrollo de la relación entre el monitor y la familia (Moraga, 2009). Esta combinación de dos metodologías diferentes en la misma intervención, (1) psicoeducación de contenido pedagógico, y (2) desarrollo de la relación monitor-cuidador, es la misma que Hebbeler y Gerlach-Downie (2002) mostraron como contradictoria en su análisis de la teoría de cambio. Nuevamente, la crítica es que, en vez de generar un modelo o teoría de cambio dirigida directamente a impactar la relación niño-cuidador, se ve al cuidador como un intermediario de las condiciones del niño. Lamentablemente, esta combinación se ve reflejada en las guías técnicas y en los objetivos específicos del programa de visitas domiciliarias que forman parte del Sistema de Protección Integral a la Infancia Chile Crece Contigo. En éstas hay un fuerte énfasis en la psicoeducación, el empoderamiento adulto y la contención emocional (Moraga, 2009).

La teoría de cambio que se esconde bajo este enfoque es que los cuidadores sólo necesitarían de las condiciones indicadas para mejorar la calidad de vida infantil. Estas condiciones se traducen en: (1) contenidos educativos e información que se entrega a través de un programa educativo, (2) empoderamiento para terminar estudios a través de apoyo social y asesoramiento, y (3) estabilidad emocional alcanzada a través del desarrollo de una relación positiva con el o la monitora. Desgraciadamente, muchas veces la falta de bienestar, salud y educación que viven los infantes es una decisión activa del cuidador que es provocada por la falta de sentido y no por la falta de condiciones o información. Justamente, las intervenciones que han enfatizado en el sentido de generar cambios en relación con un proyecto de vida han tenido impactos permanentes en el tiempo (Aracena et al., 2011).

Este artículo busca profundizar el análisis crítico del uso de la metodología pedagógica complementada con el desarrollo de la relación entre el monitor y el cuidador haciendo uso de extractos de visitas y sesiones realizadas como parte de las intervencio- 
nes de visitas domiciliarias en el programa Chile Crece Contigo. A través de estos casos se propone el uso de un método alternativo que busca generar motivación intrínseca al cambio. Este método alternativo se presenta como "Entrevista motivacional" el cual fue desarrollado por Miller y Rollnick (1999), inicialmente para el cambio en pacientes con adicciones. El objetivo de esta metodología es permitir al entrevistado encontrar sus propias motivaciones hacia el cambio, siempre desde la libertad o la empatía de elegir no querer cambiar. Paradójicamente, esta experiencia de libertad disuelve la resistencia y permite que surja una motivación genuina y profunda a implementar un cambio (Rollnick, Miller, $\&$ Butler, 2008). Este método alternativo se construye sobre una teoría diferente de cambio, en la que al cuidador se le guía para que genere su propia motivación y sentido sobre el mejoramiento de las condiciones en que se encuentran los infantes. Esto, que parece obvio tenerlo, es pasado por alto en las intervenciones mencionadas anteriormente porque, como menciona Biesta (2010), el mundo de la medicina y la salud tiene muy poca experiencia en la promoción de cambios en las personas.

\section{Chile Crece Contigo: visitas domiciliarias integrales en Chile}

Históricamente en Chile, todos los programas han compartido el objetivo de reducir el estrés familiar siendo un apoyo directo dentro del contexto del hogar de las madres (Aracena et al., 2009). Sin embargo, éstos se presentan de manera heterogénea, diferenciándose en la duración de las visitas, el tipo de visitador (profesional o paraprofesional), la población a la cual se encuentran destinados, intensidad, y su período de inicio (antes o después del parto) (Aracena et al., 2009; Gomby, Culross, \& Behrman, 1999; Sweet \& Appelbaum, 2004).

Actualmente en Chile, se realizan estudios que siguen los principios de "caja negra" planteados por Hebbeler y Gerlach-Downie (Hebbeler \& Gerlach-Downie, 2002) en el marco del Sistema de Protección Integral a la Infancia Chile Crece
Contigo. Este sistema tiene como objetivo general: "Acompañar el proceso de desarrollo de los niños y niñas que se atiendan en el sistema público de salud, desde su primer control de gestación y hasta su ingreso al sistema escolar, en el primer nivel de transición o su equivalente" (Centro de Microdatos, 2009 , p. 2). Uno de los mecanismos del sistema es el programa de Visita Domiciliaria Integral (VDI) (Kotliarenco et al., 2010). El objetivo del programa de VDI es "promover las condiciones ambientales y relacionales que favorezcan el desarrollo biopsicosocial de los niños y niñas de familias en situación de vulnerabilidad, desde el momento de la gestación hasta los seis años de edad" (Moraga, 2009, p. 26).

En términos de VDI, existen antecedentes interesantes dentro del país, como es el estudio llevado a cabo por Aracena, Krause, Pérez et al., en el cual se evalúa la intervención de visita domiciliaria de manera más positiva que el cuidado de nivel estándar entregado por los centros de salud del país, ya que promovían niveles de salud mental más elevados, mejoraban el estado nutricional entre las madres jóvenes y favorecían niveles más altos de desarrollo lingüístico en los niños. De acuerdo a Aracena y colaboradores (2011), las evaluaciones de costoefectividad de visitas domiciliarias para madres adolescentes mediante paraprofesionales han reportado resultados exitosos, en promedio, hasta los 15 meses de los niños mejorando los indicadores de salud mental y nutrición materna. Esto se condice con la política de inversión de recursos en programas destinados a madres adolescentes y sus hijos de naturaleza similar o congruente a los programas de visitas domiciliarias, los cuales han demostrado en general tener impactos positivos en la salud física y mental de las madres y en el desarrollo de sus hijos (Aracena et al., 2013).

Si bien el objetivo general del sistema y del programa de VDI está enfocado en los niños y niñas, los objetivos específicos de las VDI son diversos y no tienen como actor siempre al niño, sino muchas veces a sus padres (Cuadro 1 ). 


\section{Objetivos de la VDI para el desarrollo biopsicosocial en el periodo de gestación.}

1.1. Fomentar nutrición saludable.

1.2. Fomentar disminución/cese de consumo de tabaco, drogas, alcohol o detección de sintomatología depresiva.

1.3. Fomentar la calidad del vínculo prenatal.

1.4. Fomentar adherencia al control y educación prenatal.

1.5. Mejorar las condiciones biomédicas del niño y niña al nacer.

1.6. Fomentar la inclusión activa del padre en el apoyo a la madre, participación en controles.

1.7. Activación de redes de apoyo.

\section{Objetivos de la VDI para el desarrollo biopsicosocial en el período del puerperio y postparto.}

2.1. Fomentar lactancia exclusiva.

2.2. Fomentar la regularización de los ciclos fisiológicos del niño o niña.

2.3. Fomentar la calidad y la adaptación del ambiente del hogar para el desarrollo del niño y la niña.

2.4. Detectar factores de riesgo para la salud mental materna.

2.5. Detectar sintomatología en salud mental materna y paterna.

2.6. Fomentar la calidad de la interacción entre madre/padre y niño o niña.

2.7. Promover actividades orientadas a la activación de redes de apoyo.

2.8. Promover adaptación a rol materno y paterno con énfasis en la participación de ambos en la atención al niño o niña y en la negociación y distribución equitativa de tareas domésticas.

2.9. Fomentar la prevención del segundo embarazo en madres adolescentes.

3. Objetivos de la VDI para el desarrollo biopsicosocial en el período de infancia/crianza temprana.

3.1. Fomentar competencias de crianza del padre y de la madre.

3.2. Fomentar la participación del padre en los cuidados del niño y niña o fomentar la calidad de la relación padre y madre e hijo o hija.

3.3. Detectar interacciones vinculares inseguras entre padres y madres y sus hijos e hijas, y realizar acciones que promuevan interacciones sensibles.

3.4. Entregar conocimientos a los padres sobre desarrollo infantil integral o vigilar y fomentar el desarrollo integral del niño y niña.

3.5. Promover la estimulación psicomotora adecuada al niño y niña o fomentar la estimulación del lenguaje del niño y niña.

3.6. Fomentar el juego sensible entre padre y madre e hijo o hija, y el juego como una fuente de aprendizaje en varios ámbitos del niño; cognitivo, lenguaje, social, emocional.

3.7. Vigilar sintomatología de salud mental de la madre y del padre.

3.8. Pesquisar problemas de conducta internalizante y externalizante de niños y niñas.

3.9. Detectar y prevenir maltrato infantil, fomentar uso de técnicas de disciplina no violenta.

3.10. Prevenir el maltrato infantil y el cuidado negligente.

Fuente: Moraga, C. (Ed.) (2009). Visita domiciliaria integral para el desarrollo biopsicosocial de la infancia: Orientaciones técnicas. Santiago: Chile Crece Contigo, Sistema de Protección Integral a la Primera Infancia, Ministerio de Salud, pp. 26-27.

Es necesario aquí considerar que diversos autores han cuestionado la efectividad de los programas como modelos de intervención. Esto, ya que revisiones han llegado a concluir que los programas de visitas domiciliarias, si bien son capaces de abordar diversas problemáticas, han tenido efectos que fluctúan desde niveles pequeños a moderados y con un rango de beneficios variado (Kotliarenco et al., 2010). Por lo demás, Aracena et al. (2013) han demostrado que, en el país, las visitas domiciliarias han sido una estrategia efectiva para la promoción de la salud de las madres y los hijos a corto plazo, pero que en mediciones de seguimiento no ha sido posible encontrar los mismos resultados, situación que se encuentra en diversos estudios sobre la temática de visitas domiciliarias. Estos resultados, por lo demás, se ven influenciados por el tipo de población a la cual se dirigen, dado que dependiendo del nivel de riesgo de las familias y los objetivos de las visitas, los resultados a mediano plazo han sido 
mayores en poblaciones de menor riesgo (Aracena et al., 2013).

\section{Teoría de cambio de las visitas domiciliarias}

Considerando que las visitas domiciliarias buscan generar cambios significativos en la promoción y desarrollo de los infantes, así como en los comportamientos y habilidades parentales, es necesario evaluar la importancia de una efectiva teoría de cambio en torno a ellos. Una teoría de cambio es un conjunto de afirmaciones referidas a cómo y por qué se espera que una iniciativa funcione y que se espera que logre (Weiss, 1995). De acuerdo a Painter y colaboradores (Painter, Borba, Hynes, Mays, \& Glanz, 2008) estas teorías pueden ser usadas para explicar los determinantes estructurales y psicológicos del comportamiento y guiar el desarrollo y refinamiento de los esfuerzos por promover la salud y la educación de las personas. Entre los problemas de una falta de teoría de cambio, se encuentran: la falta de demostración de que estos programas logren efectivamente proveer un beneficio significativo o consistente en el desarrollo de los niños o en su salud, objetivo principal de la mayoría de las intervenciones (Gomby et al., 1999; Hebbeler \& Gerlach-Downie, 2002); así como también la dificultad para definir las características familiares que predicen mejores resultados, o que resultados se ajustan mejor a los diversos tipos de familias.

La prolongación de estos problemas se debe a la mantención de teorías de cambio que han resultado deficientes en la descripción de los fenómenos propios de los programas de visitas domiciliarias, o bien una falta de sistematización apropiada de los fundamentos teóricos (Kotliarenco, Gómez \& Muñoz, 2009). Tal como describen Hebbeler y Gerlach-Downie (2002), han sido explicaciones atípicas en los artículos sobre el tema para explicar el fracaso de los programas, siendo más atractivas hipótesis en la línea de una falta de implementación total, falta de cobertura o intensidad del programa. Esto es especialmente significativo si se considera que una teoría de cambio se usa para describir las metas, los métodos y los supuestos básicos en los cuales se fundamentan programas sociales como éstos, por lo que moldean los programas en sus niveles teóricos y operacionales (Hebbeler \& Gerlach-Downie, 2002). Pero, además porque, de acuerdo a la evidencia, los programas de cambio comportamental que son desarrollados de acuerdo a una teoría definida tienen una mayor probabilidad de ser exitosos (Painter et al., 2008; Raikes et al., 2014; Segal et al., 2012). De acuerdo a Raikes y colaboradores (2014), los programas de visitas domiciliarias han sido criticados por carecer de fundamento teórico y epidemiológico, así como validez, todas limitaciones que se han identificado como causas de los débiles efectos que alcanzan los programas.

¿Cuál es el mecanismo subyacente de los modelos de programa de visita domiciliaria? De acuerdo a la revisión llevada a cabo por Gomby (1999), se asume una cascada de reacciones provocadas por los programas que generen círculos virtuosos de relación padre-hijo, donde los impactos positivos iniciales en padres llevarán a efectos positivos en sus hijos en el futuro (Raikes et al., 2014). Sin embargo, los resultados sugieren que, si bien algunos padres cambian ciertas actitudes iniciales respecto al desarrollo de sus hijos, no cambian necesariamente sus comportamientos (Gomby et al., 1999). La pregunta sería entonces ¿qué se necesita para un cambio en los comportamientos de los padres?

Por otra parte, también suelen operar bajo el supuesto de que los padres son los mediadores de cambio para los hijos, ya que la mayoría de los programas han entrenado a los visitadores para interactuar directamente con los padres pero no con los niños (Raikes et al., 2014; Sweet \& Appelbaum, 2004). Esto se puede apreciar a través de los objetivos descritos para el programa de VDI y también en las recomendaciones de las acciones o intervenciones que se deben realizar durante la visita (Moraga, 2009). Raikes et al. (2014) sugieren que un programa dirigido directamente a la interacción entre el niño y los padres, donde el monitor les enseñe y modele la forma de relacionarse puede lograr impactos más fuertes. Aun así, esta forma de aproximarse a la ejecución de una visita domiciliaria aun es bastante pedagógico, en el sentido que depende de la voluntad del padre de querer, no sólo aprender, sino que llevar a cabo los cambios que requiere en su cotidianidad. Los padres pueden saber qué cambios de alimentación deben realizarse en su hogar para asegurar una alimentación a apropiada en su familia; sin embargo, por qué realizan ese cambio es algo desconocido aún por la literatura (Raikes et al., 2014).

\section{La "caja negra" de las visitas domiciliarias}

Actualmente, la VDI propone que una visita debe estar dividida en 3 momentos: (1) una fase inicial, (2) una fase de desarrollo, y (3) una fase de cierre (Moraga, 2009). La fase inicial tiene como objetivo el desarrollo de una relación significativa entre 
el agente visitador y los padres, fomentando el intercambio personal y la socialización. También se espera que el visitador pueda determinar el estado anímico de los padres y reportarlo al equipo de salud para realizar alguna intervención específica hacia los padres. En la segunda fase, se explora, evalúa y observa el ambiente en que vive el niño, la forma en que juega e interactúa con su cuidador. Se solicita una descripción de las actividades que realiza el niño o niña, por ejemplo, las horas que duerme o la alimentación. Finalmente se toman acciones o intervenciones (Cuadro 2).

Las acciones e intervenciones sugeridas, al igual que los objetivos específicos, carecen de foco. Tampoco es fácil discernir una teoría de cambio específica detrás estas acciones debido a su diversidad y a los distintos niveles que ésta representa. Este tipo de sugerencias, al ser diversas, generan y fomentan que cada visitador tenga su propia teoría de cambio a la base y emplee estas intervenciones de manera arbitraria, lo que finalmente termina reduciendo la efectividad de este tipo de programas (Hebbeler $\&$ Gerlach-Downie, 2002). Como se ha visto, la teoría de cambio es importante tanto en el diseño macro de un programa como también en sus intervenciones más acotadas. Hay otros autores (Korfmacher et al., 2008; Roggman, Cook, Peterson, \& Raikes, 2008) que ya comenzaron a trabajar sobre la aplicación de una teoría de cambio a nivel del diseño de la política pública. Por lo mismo, lo que se busca profundizar mediante este artículo es el nivel de intervenciones y acciones que se llevan a cabo durante la visita.

Recientemente se ha podido acceder a esta "caja negra" que ocurre durante la ejecución de una VDI en
Chile. Si bien existen experiencias en otros países (Hebbeler \& Gerlach-Downie, 2002), las sugerencias de este artículo buscan enriquecer el proceso de análisis de la ejecución de las visitas. Este análisis se aborda desde una teoría de cambio basada no en la pedagogía y modelamiento, sino en encontrar la motivación necesaria en la madre, el padre o el cuidador para realizar cambios que generen un impacto positivo en la salud infantil. El desafío es que no existe una propuesta activa respecto a cómo deben ser estas acciones e intervenciones, como sí las hay en una teoría de cambio basada en la entrega de conocimiento infantil.

El siguiente ejemplo es ilustrador de esta diferencia. Las acciones y estrategias para una teoría de cambio pedagógica están basadas, principalmente, en el sistema escolar. Por tanto, si la teoría de cambio indica que los impactos se producen a través de la adquisición de conocimientos, lo que debe hacer la visitadora es leer, estudiar y enseñar los contenidos de las fichas y programas. Simultáneamente se ha estudiado que la relación con la familia es importante, por tanto el agente visitador también debe preocuparse de otros contenidos no relacionados con los conocimientos que el programa desea entregar (Aracena et al., 2011), en lo posible manteniendo un equilibrio entre las acciones pedagógicas y las relacionales.

Entonces, ¿cómo podría ser este proceso si la teoría de cambio estuviese basada en hallar esta motivación en las familias para realizar sus propios cambios alineados con los objetivos del programa? Para responder esta pregunta es que se recurrirá al modelo de entrevista motivacional de Miller y Rollnick (1999). 


\section{ACCIONES O INTERVENCIONES FRECUENTES EN UNA VDI}

- Construcción de una relación de ayuda con la madre, padre y cuidadores.

- Entrega de información sobre el desarrollo del niño o niña.

- Psicoeducación sobre tópicos específicos, problemas de salud mental, etc.

- Apoyo y contención emocional, escucha activa, desarrollo de empatía.

- Identificación y comprensión de las dificultades de la familia basada en una amplia mirada de determinantes sociales, factores de riesgo, factores protectores y problemas contingentes.

- Empoderamiento de los adultos como figuras de cuidado irremplazables y únicas para su hijo o hija.

- Fomento de esperanza y ayudar a construir alternativas de solución a las dificultades.

- Detección de los peligros de seguridad física del medioambiente y fomentar la prevención de accidentes en el hogar.

- Otorgar apoyo instrumental a través de información actualizada, concreta y contingente sobre la oferta de los servicios sociales de su comunidad.

- Realizar intervención en crisis cuando sea necesario.

- Fomento de la calidad y seguridad de la relación o interacción padre, madre y cuidadores e hijo o hija.

- Fomento, psicoeducación, o modelamiento de juego interactivo y estimulante entre adultos y niños o niñas.

- Fomento y estimulación de la participación activa y temprana del padre en los cuidados básicos del niño o niña y en las labores del hogar.

- Fomento de la expresión de apoyo emocional del padre hacia la madre durante la gestación y crianza.

- Fomento del nivel de reflexión de madres y padres y cuidadores sobre los sentimientos y pensamientos del niño o niña que ayuden a ampliar la comprensión de sus conductas y dificultades.

- Integración y fomento del uso de materiales de juego, apoyo y educación entregados por Chile Crece Contigo.

- Fortalecimiento de la detección por parte de los adultos de situaciones de vulnerabilidades que puedan estar presentes en la familia y trabajar en la superación progresiva de éstos.

- Fomentar lactancia exclusiva.

- Conexión y motivación de la familia en la participación de prestaciones universales de salud del Chile Crece Contigo.

- Apoyo individual y específico a la mujer víctima de violencia de género.

- Apoyo especial y continuado a familias que presentan maltrato infantil.

- Apoyo especial para la mujer gestante con factores de riesgo.

- Apoyo y refuerzo específico de niños y niñas con retraso en el desarrollo psicomotor (lenguaje, físico, social).

- Detección precoz de otro tipo de riesgos observados en el hogar.

Fuente: Moraga, C. (Ed.). (2009). Visita domiciliaria integral para el desarrollo biopsicosocial de la infancia: Orientaciones técnicas. Santiago: Chile Crece Contigo, Sistema de Protección Integral a la Primera Infancia, Ministerio de Salud, pág. 34.

\section{La entrevista motivacional}

A raíz de su trabajo en adicciones, Miller y Rollnick (1999) plantean una forma de entrevista, centrada en el cliente, para incentivar o hacer de primer paso dentro de un proceso terapéutico. A medida que pasan los años, esta metodología de entrevista se ha aplicado en diversos contextos y ha probado que es una técnica efectiva que ayuda a las personas a cambiar (Rollnick et al., 2008). En años posteriores al desarrollo de esta metodología, los autores han abogado por su uso en salud pública afirmando que: "La mayoría de los malestares que llevan a la gente a consultar por profesionales de la salud (e.i. médicos, dentistas, enfermeras, quiroprácticos) son, en gran parte, prevenibles o remediables a través de un cambio en la conducta de salud (...) En el siglo 21, la salud se trata de la administración de condiciones a largo plazo que necesitan de cambios en la conducta de salud (...) Sin embargo, la mayoría de las personas que buscan mejorar su salud todavía están buscando una cura médica" (Rollnick et al., 2008, pp. 3-4).

Los autores describen la técnica de la siguiente manera: "Se trata de una forma concreta de ayudar a 
las personas para que reconozcan y se ocupen de sus problemas potenciales y presentes (...) Cuando este enfoque se aplica adecuadamente, el paciente presenta las razones para cambiar y no el terapeuta" (Miller et al., 1999, p. 80). Ahora, esta entrevista no debe ser confundida con una terapia ya que, a diferencia de un ambiente terapéutico completamente centrado en y desde el cliente, la entrevista motivacional actúa con una idea clara de objetivo y tiempos a la hora de intervenir.

A pesar de tener esta claridad, los autores son claros en especificar que no forma parte de la entrevista motivacional: (1) no es un cuestionario médico, (2) no es una confrontación, (3) no son consejos desde un experto, (4) tampoco es una etiquetación de la condición o situación, (5) no tiene un énfasis prematuro y (6) tampoco trabaja sobre la culpa del cliente. Por el contrario, lo que busca la entrevista motivacional es: (1) la realización de preguntas abiertas, (2) escuchar activamente al cliente, (3) afirmar, (4) resumir, (5) provocar afirmaciones automotivadoras. Esta última estrategia es la que produce y direcciona la motivación hacia el objetivo del programa. "Las cuatro estrategias anteriores son fundamentales para la entrevista motivacional. Sin embargo, si éstas fueran las únicas estrategias utilizadas sería bastante fácil quedar bloqueado en medio de la ambivalencia" (Miller et al., 1999, p. 110).

En resumen, si las primeras 4 estrategias tienen como objetivo que surja la motivación intrínseca del cliente, esta última es la que orienta y permite al monitor o agente visitador ir avanzando en la entrevista. Como se menciona anteriormente, en la entrevista motivacional se espera que el cliente, en este caso la familia, sea el que presente las razones y argumentos para cambiar. Se da vuelta la conversación donde, en vez de que el visitador sea el que empuje por los cambios, sea la madre la que argumente sobre la posición de cambiar. Para lograr estas afirmaciones automotivadoras se propone: (1) el uso de preguntas evocadoras, (2) plantear un balance decisional, (3) detallar o elaborar la situación que se quiere cambiar, (4) utilizar los extremos, (5) mirar hacia atrás en busca de situaciones donde lo que se quiera cambiar sea diferente (6) mirar hacia delante para imaginar un momento en que la situación efectivamente haya cambiado, (7) analizar los objetivos, y (8) crear paradojas donde el entrevistador plantee lo que se necesita cambiar desde la ausencia. Finalmente, estas cinco estrategias se resumen en cinco principios generales de la entrevista motivacional:

\section{Expresar empatía}

\section{Desarrollar la discrepancia}

3. Evitar la discusión

4. Deslizarse en la resistencia

\section{Fomentar la autoeficacia}

Bajando la entrevista motivacional a elementos concretos de un programa de visita domiciliaria integral, se han agrupado estos 5 principios en dos tipos de intervenciones que dan cuenta de las acciones y estrategias que pueden adoptar los agentes visitadores. El primer tipo de intervenciones agrupa los principios de expresar empatía, evitar la discusión y deslizarse en la resistencia. Tal como explican Miller y Rollnick, (1999), los primeros 4 principios y estrategias buscan dar la oportunidad para que se despliegue la motivación auténtica del entrevistado, en este caso de la madre, el padre o el cuidador. Este es el tipo de intervenciones que buscan la apertura sin juicios ni defensas sobre la situación que vive la familia. En la práctica, estas acciones corresponden a fomentar que la conversación siga de manera fluida mediante la realización de preguntas abiertas, escuchar, afirmar, resumir. En ningún momento plantear o imponer.

El segundo tipo de intervenciones corresponden a los principios de fomentar la autoeficacia y desarrollar la discrepancia. La estrategia de este principio es que la familia haga afirmaciones automotivadoras respecto a la situación que desea cambiar. Como se listó anteriormente, las técnicas para promover las afirmaciones automotivadoras son múltiples y merecen un tratamiento independiente. En este artículo se han destacado viñetas representativas de intervenciones que van en la dirección opuesta a la entrevista motivacional y para cada uno de estos dos tipos de intervención. El objetivo es ejemplificar y mostrar cómo los agentes visitadores utilizan estas estrategias de manera natural, sin entrenamiento previo con respecto a la entrevista motivacional.

\section{Dirigir: intervenciones que van en la dirección opuesta a generar motivación}

En general, el error de este tipo de intervenciones es que la persona que hace la visita domiciliaria impone o sugiere un tema de forma radical sin prestar atención a la línea discursiva que se venía desarrollando. En este estilo de intervenciones, el monitor o visitador se "hace cargo" y se plantea desde una relación desigual con respecto al conocimiento, experticia, autoridad o poder. Muchas veces esta forma de comunicarse salva vidas en salud y, en esencia, un director es quien dice lo que hay que 
hacer. Sin embargo, a la hora de generar cambios en el estilo de vida, las respuestas estarán siempre en el cliente.

Dentro de la definición de "que no forma parte de una entrevista motivacional" se mencionan: (1) cuestionario médico, (2) confrontación, (3) consejos desde un experto, (4) etiquetación de la condición o situación, (5) énfasis prematuro y (6) culpa de la madre. Cualquiera de estas acciones tiene como punto en común que el agente visitador impone su posición sobre la madre o el padre.

Ejemplo 1: [Confrontación, consejos, énfasis prematuro]

Visitadora: ¿Algún tema que te interese, que quisierai que lo habláramos ahora?

Madre: No sé...Cualquier tema, po'

V: No sé, po', dime tú... la alimentación y postparto, actividades físicas para una embarazada, prevención de enfermedades de transmisión sexual, controles médicos, síntomas de alarma que requieren acudir al centro de salud, aprender a decir que no, divertirse para sentirse bien, higiene, el bebé de cero a tres meses, aprendiendo a ver televisión, una buena alimentación, el derecho de tener una pareja, planificación de otros embarazos, del presupuesto, planificando el tiempo para estudiar, en búsqueda del trabajo, vecinos y vecinas: una posibilidad de ayuda, encontrando ayuda para resolver las dificultades, teléfonos de emergencia, ser mujer y madre, ser buena mamá, los ajustes cuando llega el bebé, la relación con mi mamá, la importancia de las abuelas y los abuelos, paternidad, bolso para el día de nacimiento de la guagua, preparando mi currículum y... amigos y amigas. ¿Cuál crees tú que te llama un poco la atención?

M: El último, el bolso.

$\mathrm{V}$ : ¿El bolso? (Busca hoja) El bolso para el día del nacimiento. Eso ya lo tienes que tenerlo visto como a las 37 semanas, 36 semanas, ya tienes que tener listo tu bolso para irte al hospital. (Lee) En el bolso deber llevar para ti: cami- sa de dormir, zapatillas de levantarse, toalla higiénica, los artículos de aseo: jabón, champú y pasta de dientes, ¿ya? Eh... el carnet de identidad, credencial de salud y el carnet maternal. Eso es lo primordial. Y para tu hijo, para la primera, o sea cuando ya estai en la sala de parto ya, lo primordial es: un pilucho body, osito, unas panties, un par de calcetines, una camiseta, el gorro de algodón, cuatro pañales desechables, iya? Eso es con lo primordial que tu necesitai cuando recién te vayas a mejorar, porque después tu marido puede entrar todo el día, hasta las 7 de la tarde, y él te puede estar llevando las cosas, llevar las otras cosas que necesitas, porque entre más chico el bolso es mejor, porque hay personas que llevan unos tremendos bolsos y todo al tiro; a veces se extravían, a veces no saben pa' dónde los mandan, o es tan chico el espacio que tienen adentro que no... no pueden guardarlos bien, po'. Así que ese es el bolso que necesitas cuando... cuando recién te vayas a mejorar, iya? ¿Alguna duda?

M: No...

V: Es calladita (ríe) ¿Alguna otra duda? M: No

V: ¿Alguna otra pregunta?

M: No

$\mathrm{V}$ : ¡¿Cómo no va a querer saber nada?!

M: (Ríe) Es que no... no tengo dudas.

$\mathrm{V}$ : No tienes dudas, tienes todo bien claro. ¿Los embarazos que tuviste cómo han sido?

M: Buenos.

Ejemplo 2 [listado médico sin prestar atención a la situación]:

Visitadora...vamos a recorrer el nacimiento del niño.

Madre: Ya.

V: Ya. Tu pareja ¿cómo se llama tu pareja?

M: Luis León. 
V: Luis León. El niño nació el 7 de febrero, icierto?

M: Sí.

V: Ya. Mira, nosotros estamos aquí para informarte sobre lo que tú necesites, las necesidades tuyas.

M: Ya.

V: ¿Ya?. Tu relación con tu pareja, con tu familia.

M: Ya.

V: Ya. Lo que a ti te incomode... Eh, y así va a ir surgiendo todo...

Lamentablemente, este tipo de intervenciones son las más comunes dentro de las visitas revisadas. Este tipo de acciones cortan y limitan el diálogo, y no permiten que la motivación de la madre pueda surgir

Seguir: Expresar empatía + Deslizarse en la resistencia + Evitar la discusión

Son las intervenciones dónde el monitor toma y continúa la conversación a partir de lo que expresa la madre. Se puede tomar incluso de lo último que se dijo. En este tipo de intervenciones el foco es que el sujeto siga conversando sobre lo que estaba diciendo y cómo promover esa conversación. En su Manual para Entrevista Motivacional en Atenciones de Salud, Rollnick et al. (2008) explican que estas intervenciones se caracterizan por seguir al cliente, en este caso la madre. Si bien en el primer estilo se informa y pregunta, pero no se escucha, este segundo estilo consiste justamente en escuchar y preguntar, pero no necesariamente informar.

Ejemplo 1: Madre: Ojalá, en realidad cuando en el único momento en que estoy tranquila es cuando no está él, en realidad. Cuando está en el colegio, ahí como que estoy relajada, hago las cosas bien, lo veo a él tranquilo. Pero cuando está aquí como que no, de repente lo toma y lo hace así (hace gesto de sacudir), (baja la voz) como que lo quiera dañar, claro, porque llegó y lo sacó del coche entonces yo le digo, "no, déjalo ahí un ratito tranquilo". Entonces, en realidad está él, como que yo me, mi suegra endenante cuando llegamos yo venía, entre a la tienda y como que todo me, no hallaba qué hacer. Empecé a mirar y como que, nerviosa no sé por qué, y empecé a sudar así, estaba toda mojada. Y como que todo, empecé a mirar todo y como que no hallaba, no hallaba en realidad pa' dónde mirar, estaba como nerviosa.

Visitadora: Y, estaba ahí pero no veiai.

M: Claro, como que no sé, y primera vez que me pasa, como que nunca me había pasado.

Ejemplo 2: Madre: También tengo hermanos pero tampoco los veo. O sea yo soy sola aquí, no, no tengo eh... familia así cercana que me visite, mis hermanos, mi mamá, no, nadie. Yo soy yo, y con mi fam, y con el papá de él, mi hijo.

Visitadora: Esa es tu familia. (Estrategia de Resumen)

M: Claro. No, de parte de mi mamá no, no. Y mi papá, poh.

$\mathrm{V}$ : ¿Y hace mucho años se rompió esa ese qué? (Pregunta abierta)

M: Hace... A ver, fue como... a ver, yo le voy a contar toda la historia porque.... A los nueve años yo me fui con mi papá cuando se separaron ya, nueve años me fui con mi papá. Eh, a los doce, hasta los doce años estuve con él, porque mi ¿papá? era alcohólico, me dejaba sola en la casa, entonces una vecina llamó a una asistente social y ellos me llevaron a una casa acogida. E igual ahí me acuerdo porque era en Avenida Matta. Después de esa casa acogida, ehm, me fueron a dejar a un internado, pero mi mamá nunca se preocupó como por mí por irme a ver, para saber de mí, nada. Mi papá tampoco ese tiempo. Entonces, yo lo conocí, conocí a mi pareja cuando tenía catorce años... eh, quedé embaraza y él me sacó del internado. Y... acá ahora estoy con él po, ja ja.

El objetivo de estas intervenciones es, simplemente, fomentar el diálogo. Como se describió anteriormente, la diferencia entre la entrevista motivacional y una conversación abierta es que existe un objetivo claro y definido que se quiere alcanzar mediante esta intervención. Es por esto que seguir al cliente es importante; sin embargo, también es necesario hacer de guía y acompañarlo una vez que haya decidido hacia dónde desea ir. 
Guiar: Desarrollar la discrepancia + Fomentar la autoeficacia

Son las intervenciones más desafiantes para un monitor sin entrenamiento. Para realizarlas, el entrevistador debe tener entendimiento del sentido general que tiene la conversación y hacia dónde apunta la motivación de los entrevistados. Los autores explican este estilo mediante la metáfora de guiar. El cliente es quien debe decidir a dónde quiere ir, y para eso contrata un guía o un agente de viajes para que le ayude a llegar a su destino. Este estilo mezcla de manera equitativa el escuchar, el preguntar y el informar, de manera simultánea. Estas son las intervenciones más representativas de la entrevista motivacional

Rollnick et al. (2008) resumen estos tres estilos de la siguiente manera: "un padre directivo le dice a su hijo lo que tiene que hacer paso a paso y corrige sus errores, incluso puede tomar el lugar del niño y decirle 'déjame hacerlo'. El padre seguidor se sienta atrás y mira al niño intentar bajo ensayo y error sin ofrecer su ayuda. El padre que guía hace un poco de los dos, mira pacientemente y con interés, pero también se deja caer de vez en cuando y susurra en el oído del niño 'tal vez podrías intentar comenzar con los bloques más grandes', se retira y lo deja intentar" (p. 16).

De esta manera puede generar afirmaciones automotivantes, donde la madre o el padre sean quienes dan la argumentación para cambiar.

Ejemplo 1: Madre: Es como yo a mi hija de repente le digo 'ya, mi amor, ordena tus juguetes', y a él yo le tengo que decir 'ya podí ver al Aldo, podí llevar a la Ema al baño, podí', así es una... ah, ya me aburrió, así que ya no lo pesco.

Visitadora: ¿O sea no se le ocurre a él solo? (Pregunta evocadora)

M: Noo, es como no sé, como medio pavo.

\section{V: Ajajaja.}

M: Lo dejo ya porque antes terminaba peleando todo el día con el así, y a mí sí que me da la tontera, así que...

V: Y él... pero yo me acuerdo que la sesión pasada algo me dijiste que sí te ayudaba harto él.

M: Si, me ayudaba harto, pero como que de un tiempo a otro empezó así como a ponerse más pesadito así.
V: Ya.

M: Y ya, no sé, ya me está aburriendo.

V: Ajaja.

M: Sí, de repente me dan ganas de irme de aquí así, escapar lejos así, oh, no sé, cambiaron tanto las cosas. Cambió muy rápido, muy drástico todo así, así que no sé, de repente me dan ganas de decir, ah, ya sé, pescar todas mis cosas, mis hijos, e irme y decirle ya shao, dejarlo solo un rato... Tengo tantas ganas de escaparme así o tener un día sola así. (Afirmación automotivante)

V: Un día libre para ti.

Este último tipo de intervenciones es la más escasa dentro del material revisado. Esto es normal, ya que en el modelo actual de intervención no se contempla un entrenamiento en entrevista motivacional. Aun así, se puede apreciar que la implementación de este modelo es natural, no requiere de un lenguaje sofisticado, sino todo lo contrario, se requiere estar conectado con la realidad que está viviendo el entrevistado. Esto es lo que Miller y Rollnick (1999) llaman expresar empatía.

\section{Conclusiones}

Actualmente, el modelo de visita domiciliaria integral que forma parte del Sistema de Protección Integral a la Infancia Chile Crece Contigo no cuenta con un modelo explicativo o teoría de cambio claro. Cuenta con una diversidad de estrategias que se enfocan en distintos actores. Si bien existe un acuerdo en la literatura respecto al potencial de este tipo de programas, las investigaciones muestran que el impacto es limitado en cuanto no exista un objetivo claro y una teoría de cambio que se ajuste y esté anclada en la interacción que se produce entre la madre, el padre o el cuidador con el infante. Este cambio en la interacción no es fácil de producir y se ha intentado fomentar el modelamiento de las conductas parentales durante las visitas domiciliarias con una efectividad limitada. La entrevista motivacional ha probado ser una alternativa exitosa para producir cambios en las personas en intervenciones sociales relacionadas con otros factores de vulnerabilidad. Implementar la entrevista motivacional requiere de capacitación y formación específica. Esta capacitación no es mayor a la que se necesita para dominar los contenidos que se esperan impartir a través del programa. En este artículo se muestran viñetas de ejemplos entre agentes visitadores no 
entrenados en entrevista motivacional. El desafío futuro es probar y validar a través de grabaciones las diferencias que se producen en la interacción, relación, aprendizajes y cambios utilizando estas estrategias. Analizar diferentes estrategias de visitas domiciliarias a través del prisma de "la apertura de la caja negra" es el principal desafío para el logro de mejores resultados en visitas domiciliarias.

\section{Referencias}

ARACENA, M., KRAUSE, M., PEREZ, C., MENDEZ, M. J., SALVATIERRA, L., SOTO, M., PANTOJA, T., et al. (2009). A cost-effectiveness evaluation of a home visit program for adolescent mothers. Journal of Health Psychology, 14(7), 878-887.

ARACENA, M., KRAUSE, M., PÉREZ, J. C., BEDREGAL, P., UNDURRAGA, C. \& ALAMO, N. (2013). Efectos de mediano plazo de un programa de intervención para madres adolescentes. Atención Primaria, 45(3), 157-164.

ARACENA, M., LEIVA, L., UNDURRAGA, C., KRAUSE, M., PEREZ, C., CUADRA, V., SILVIA CAMPOS, M. et al. (2011). Effectiveness of a home visit program for adolescent mothers and their children. Revista Medica De Chile, 139(1), 60-65.

BIESTA, G. (2010). Why "What Works" Still Won't Work: From Evidence-Based Education to Value-Based Education. Studies in Philosophy \& Education, 29(5), 491-503. http://doi.org/10.1007/s11217-010-9191-x

CENTRO DE MICRODATOS. (2009). Diseño Evaluación de Programas Nuevos:Subsistema de Protección Integral a la Infancia Chile Crece Contigo.

GOMBY, D. S. (2007). The promise and limitations of home visiting: Implementing effective programs. Child Abuse \& Neglect, 31(8), 793-799.

GOMBY, D. S., CULROSS, P. L., \& BEHRMAN, R. E. (1999). Home Visiting: Recent Program Evaluations: Analysis and Recommendations. The Future of Children, 9(1), 4.

HEBBELER, K. M. \& GERLACH-DOWNIE, S. G. (2002). Inside the black box of home visiting: a qualitative analysis of why intended outcomes were not achieved. Early Childhood Research Quarterly, 17(1), 28-51.

KENDRICK, D. (2000). Does home visiting improve parenting and the quality of the home environment? A systematic review and meta analysis. Archives of Disease in Childhood, 82(6), 443-451. BMJ Publishing Group Ltd and Royal College of Paediatrics and Child Health.

KORFMACHER, J., GREEN, B., STAERKEL, F., PETERSON, C., COOK, G. A., ROGGMAN, L. A., FALDOWSKI, R. A. et al. (2008). Parent Involvement in Early Childhood Home Visiting. Child \& Youth Care Forum, 37(4), 171-196.
KOTliARENCO, M. A., GÓMEZ, E. \& MUÑOZ, M. (2009). Centros de desarrollo infantil en Chile: Una experiencia piloto (No. 8). Perspectivas CEES.

Kotliarenco, M. A., Gómez, E., Muñoz, M. M. \& Aracena, M. (2010). The characteristics, effectiveness and challenges of home visiting in early intervention programmes. Revista de Salud Pública, 12(2), 184-196. Instituto de Salud Publica, Faculdad de Medicina Universidad Nacional de Colombia. Retrieved from http://www.scielosp.org/scielo.php? pid=S012400642010000200002 \&script=sci_arttext

LeCroy, C. W., \& Whitaker, K. (2005). Improving the quality of home visitation: An exploratory study of difficult situations. Child Abuse \& Neglect, 29(9), 1003-1013

Miller, W. R., Rollnick, S. \& Pociello, A. T. (1999). La entrevista motivacional: preparar para el cambio de conductas adictivas. Paidós.

Moraga, C. (Ed.). (2009). Visita domiciliaria integral para el desarrollo biopsicosocial de la infancia: Orientaciones técnicas. Santiago: Chile Crece Contigo, Sistema de Protección Integral a la Primera Infancia, Ministerio de Salud.

Painter, J. E., Borba, C. P. C., Hynes, M., Mays, D., \& Glanz, K. (2008). The Use of Theory in Health Behavior Research from 2000 to 2005: A Systematic Review. Annals of Behavioral Medicine, 35(3), 358-362.

Raikes, H. H., Roggman, L. A., Peterson, C. A., BrooksGunn, J., Chazan-Cohen, R., Zhang, X. \& Schiffman, R. F. (2014). Theories of change and outcomes in home-based Early Head Start programs. Early Childhood Research Quarterly, 29(4), 574-585.

Roggman, L. A., Cook, G. A., Peterson, C. A. \& Raikes, H. H. (2008). Who Drops Out of Early Head Start Home Visiting Programs? Early Education and Development, 19(4), 574-599.

Rollnick, S., Miller, W. R., \& Butler, C. (2008). Motivational interviewing in health care: helping patients change behavior. New York: Guilford Press.

Segal, L., Sara Opie, R. \& Dalziel, K. (2012). Theory! The Missing Link in Understanding the Performance of Neonate/Infant Home-Visiting Programs to Prevent Child Maltreatment: A Systematic Review. Milbank Quarterly, 90(1), 47-106. Blackwell Publishing Inc.

Sweet, M. A. \& Appelbaum, M. I. (2004). Is Home Visiting an Effective Strategy? A Meta-Analytic Review of Home Visiting Programs for Families With Young Children. Child Development, 75(5), 1435-1456.

Weiss, C. H. (1995). Nothing as practical as good theory: Exploring theory-based evaluation for comprehensive community initiatives for children and families. In J. P. Connell, A. C. Kubisch, L. B. Schorr \& C. H. Weiss (Eds.), New Approaches to Evaluating Community Initiatives: Concepts, Methods, and Contexts (pp. 6592). Queenstown, MD: Aspen Institute. 